\title{
Characterization of Associative PU-algebras by the Notion of Derivations
}

\author{
Mehmood Khan, Dawood Khan, Khalida Mir Aalm \\ Department of Mathematics, University of Balochistan, Quetta, Pakistan \\ Email address: \\ dawooddawood601@gmail.com (M. Khan),Mehmud412@gmail.com (D. Khan), Khalidamir098@gmail.com (K. M. Aalm)
}

\section{To cite this article:}

Mehmood Khan, Dawood Khan, Khalida Mir Aalm. Characterization of Associative PU-algebras by the Notion of Derivations. American Journal of Mathematical and Computer Modelling. Vol. 6, No. 1, 2021, pp. 14-18. doi: 10.11648/j.ajmcm.20210601.13

Received: January 18, 2021; Accepted: February 2, 2021; Published: March 4, 2021

\begin{abstract}
In this manuscript we insert the concept of derivations in associative PU-algebras and discuss some of its important results such that we prove that for a mapping being a (Left, Right) or (Right, Left)-derivation of an associative PUalgebra then such a mapping is one-one. If a mapping is regular then it is identity. If any element of an associative PU-algebra satisfying the criteria of identity function then such a map is identity. We also prove some useful properties for a mapping being (Left, Right)-regular derivation of an associative PU-algebra and (Right, Left)-regular derivation of an associative PUalgebra. Moreover we prove that if a mapping is regular (Left, Right)-derivation of an associative PU-algebra then its Kernel is a subalgebra. We have no doubt that the research along this line can be kept up, and indeed, some results in this manuscript have already made up a foundation for further exploration concerning the further progression of PU-algebras. These definitions and main results can be similarly extended to some other algebraic systems such as BCH-algebras, Hilbert algebras, BFalgebras, J-algebras, WS-algebras, CI-algebras, SU-algebras, BCL-algebras, BP-algebras and BO-algebras, Z- algebras and so forth. The main purpose of our future work is to investigate the fuzzy derivations ideals in PU-algebras, which may have a lot of applications in different branches of theoretical physics and computer science.
\end{abstract}

Keywords: PU-Algebras, (Left, Right)-derivations of PU-algebras, (Right, Left)-derivations of PU-algebras, Regular Derivations of PU-algebras.

\section{Introduction}

In 1966, Y. Imai and K. Isèki introduced two classes of abstract algebras: BCK-algebras and BCI-algebras [1-3]. It is known that the class of BCK-algebras is a proper subclass of the class of BCI-algebras. Neggers et al. [4] introduced a notions, called Q-algebras, which is a generalization of BCH / BCI / BCK-algebras and generalized some theorems discussed in BCIalgebras. Megalai and Tamilarasi [5] laid down the foundation of a notion, called TM-algebra. Moreover, Mostafa et al. [6] introduced a new algebraic structure called PU-algebra, which is a dual for TM-algebra and investigated severed basic properties. Moreover they derived new view of several ideals on PUalgebra and studied some properties of them. Derivation is a very interesting and important area of research in the theory of algebraic structures in mathematics. Several authors [7-11] have studied derivations in rings and near rings. Jun and Xin [12] applied the notions of derivations in ring and near-ring theory to BCI-algebras, and they also introduced a new concept called a regular Derivation in BCI-algebra. They investigated some of its properties, defined a d -derivations ideal and gave conditions for an ideal to be d-derivations. Later, Abujabal and Al-Shehri [13], defined a left derivations in BCI-algebras and investigated a regular left derivations. Zhan and Liu [14] studied f-derivations in BCI-algebras and proved some results. Muhiuddin and Alroqi $[15,16]$ introduced the notions of $(\alpha, \beta)$-derivations in a BCI-algebras and investigated related properties. They provided a condition for a $(\alpha, \beta)$ - derivations to be regular. They also introduced the concepts of a $\mathrm{d}(\alpha, \beta)$ - invariant $(\alpha, \beta)$ derivations and $\alpha$-ideal, and then they investigated their relations. Furthermore, they obtained some results on regular $(\alpha, \beta)$ derivations. Moreover, they studied the notions of t-derivations on BCI-algebras [17] and obtain some of its related properties. Further, they characterized the notions of p-semisimple BCIalgebras $\mathrm{X}$ by using the notions of t-derivations. Abujabal and Shehri in their pioneer paper [18], defined the derivations as, for a self-map, $d$, for any algebra $\mathrm{X}, d$ is a left-right derivation (briefly $(l, r)$-derivation) of $\mathrm{X}$ if it satisfies the identity $d(\mathfrak{a} *$ $\mathfrak{b})=(d(\mathfrak{a}) * \mathfrak{b}) \wedge(\mathfrak{a} * d(\mathfrak{b}))$. For all $\mathfrak{a}, \mathfrak{b} \in X$. If $d$ satisfies the identity $d(\mathfrak{a} * \mathfrak{b})=(\mathfrak{a} * d(\mathfrak{b})) \wedge(d(\mathfrak{a}) * \mathfrak{b})$ for all $\mathfrak{a}, \mathfrak{b} \in X$, 
then $d$ is a right-left derivation (briefly $(r, l)$-derivation) of $\mathrm{X}$. If $d$ is both $(l, r)$ - derivation and $(r, l)$-derivation, then $d$ is a derivation of $\mathrm{X}$. The aim of the paper is to complete the studies on PU-algebra; in particular, we aim to apply the notion of derivation on associative PU-algebra and obtain some related properties. We start with definitions and propositions on PUalgebra taken from [6].

Then, we redefine the notion of derivation in associative PU-algebra and prove that for $\phi$ being a (Left, Right) or (Right, Left) -derivation of an associative PU-algebra $Z$ then $\phi$ is one-one map. If $\phi$ is a regular map then it is identity. If there exists an element $\mathfrak{a} \in Z$, such that $\phi(\mathfrak{a})=\mathfrak{a}$ then the map $\phi$ is identity. We prove that If $\phi$ is (Left, Right) -regular derivation of $Z$ then $\phi(\mathfrak{a})=\mathfrak{a} \wedge \phi(\mathfrak{a})$ also if $\phi$ is (Right, Left)-regular derivation of $Z$ then $\phi(\mathfrak{a})=\phi(\mathfrak{a}) \wedge \mathfrak{a}$, $\forall \mathfrak{a} \in Z$. We prove that if $\phi$ is a self-map of an associative PU-Algebra $Z$ then $(\mathfrak{a} *(\mathfrak{a} * \phi(\mathfrak{a}))) * \mathfrak{a}=(\phi(\mathfrak{a}) *(\phi(\mathfrak{a}) *$ $\mathfrak{a})) * \mathfrak{a}$. We also prove that if $\phi$ is a regular (Right, Left)derivation of an associative PU-algebra $Z$ then $\operatorname{Ker}(\phi)=\{\mathfrak{a} \in$ $Z: \phi(\mathfrak{a})=0\}$ is a subalgebra of $Z$.

\section{Preliminaries}

This section consists of some preliminary definitions and basic facts about PU-algebra which are useful in the proofs of our results. Throughout this research work we denote the PUalgebra always by $Z$.

Definition 2.1: [6] PU-algebra $(Z, *, 0)$ is a class of the type $(2,0)$ algebras which satisfies the $\left(\mathrm{P}_{1}\right)$ and $\left(\mathrm{P}_{2}\right)$ conditions for all $\mathfrak{p}, \mathfrak{q}, \mathfrak{r} \in Z$, where

$\left(\mathrm{P}_{1}\right) 0 * \mathfrak{a}=\mathfrak{a}\left(\mathrm{P}_{2}\right)(\mathfrak{a} * \mathfrak{c}) *(\mathfrak{b} * \mathfrak{c})=\mathfrak{b} * \mathfrak{a}$

While the binary relation ' $\leq$ ' on $Z$ is defined as $\mathfrak{a} \leq \mathfrak{b} \Leftrightarrow$ $\mathfrak{b} * \mathfrak{a}=0$.

Proposition 2.2: [6] In PU-algebra $(Z, *, 0)$ the following results are true for all $\mathfrak{a}, \mathfrak{b}, \mathfrak{c} \in Z$.

$\left(\mathrm{P}_{3}\right) \mathfrak{a} * \mathfrak{a}=0$

$\left(\mathrm{P}_{4}\right)(\mathfrak{a} * \mathfrak{c}) * \mathfrak{c}=\mathfrak{a}$

$\left(\mathrm{P}_{5}\right) \mathfrak{a} *(\mathfrak{b} * \mathfrak{c})=\mathfrak{b} *(\mathfrak{a} * \mathfrak{c})$

$\left(\mathrm{P}_{6}\right) \mathfrak{a} *(\mathfrak{b} * \mathfrak{a})=\mathfrak{b} * 0$

$\left(\mathrm{P}_{7}\right)$ The following three results are similar in $(Z, *, 0)$

(1): $\mathfrak{b}=\mathfrak{c}(2): \mathfrak{b} * \mathfrak{a}=\mathfrak{c} * \mathfrak{a}(3): \mathfrak{a} * \mathfrak{b}=\mathfrak{a} * \mathfrak{c}$

$\left(\mathrm{P}_{8}\right)$ Both (left and right) cancellation properties hold in $(Z, *, 0)$.

Definition 2.3: [6] PU-algebra $(Z, *, 0)$ is said to be associative if it satisfies the condition $\mathfrak{a} *(\mathfrak{b} * \mathfrak{c})=(\mathfrak{a} * \mathfrak{b}) * \mathfrak{c}$ for all $\mathfrak{a}, \mathfrak{b}, \mathfrak{c} \in Z$.

\section{Main Results}

Definition 3.1:- Let $(Z, *, 0)$ is an associative PU-algebra and $\phi: Z \rightarrow Z$ is a self-map then $\phi$ called (Left, Right) derivation on $Z$ if $\phi(\mathfrak{a} * \mathfrak{b})=(\phi(\mathfrak{a}) * \mathfrak{b}) \wedge(\mathfrak{a} * \phi(\mathfrak{b}))$.

Definition 3.2:- Let $(Z, *, 0)$ is an associative PU-algebra and $\phi: Z \rightarrow Z$ is a self-map then $\phi$ is called (Right, Left)derivation on $Z$ if $\phi(\mathfrak{a} * \mathfrak{b})=(\mathfrak{a} * \phi(\mathfrak{b})) \wedge(\phi(\mathfrak{a}) * \mathfrak{b})$.

Definition 3.3: If $\phi$ is both (Left, Right)-derivation and (Right, Left)-derivation on $Z$, then $\phi$ is called derivation on $Z$. Definition 3.4: A self-map $\phi: Z \rightarrow Z$ on associative PU- algebra $Z$ is called regular if $\phi(0)=0$.

Example 3.5: Let the set $Z=\{0, \mathfrak{a}, \mathfrak{b}, \mathfrak{c}\}$ defined by the following table.

Table 1. Tabular arrangement of the values of the set satisfying the axioms of associative $P U$-algebra.

\begin{tabular}{lllll}
\hline$*$ & $\mathbf{0}$ & $\mathbf{a}$ & $\mathbf{b}$ & $\mathfrak{c}$ \\
\hline 0 & 0 & $\mathfrak{a}$ & $\mathfrak{b}$ & $\mathfrak{c}$ \\
$\mathfrak{a}$ & $\mathfrak{a}$ & 0 & $\mathfrak{c}$ & $\mathfrak{b}$ \\
$\mathfrak{b}$ & $\mathfrak{b}$ & $\mathfrak{c}$ & 0 & $\mathfrak{a}$ \\
$\mathfrak{c}$ & $\mathfrak{c}$ & $\mathfrak{b}$ & $\mathfrak{a}$ & 0 \\
\hline
\end{tabular}

Is an associative PU-algebra and a map, $\phi: Z \rightarrow Z$ defined by

$\phi(0)=\mathfrak{c} \phi(\mathfrak{a})=\mathfrak{b} \phi(\mathfrak{b})=\mathfrak{a}$ and $\phi(\mathfrak{c})=0$ is both (Left, Right) derivation and (Right, Left) -derivation on $Z$ and thus a derivation of $Z$.

Proposition 3.6: Let $\phi$ be a (Left, Right)-derivation of an associative PU-algebra $Z$ then

$\left(\mathrm{P}_{9}\right): \phi(0)=\phi(\mathfrak{a}) * \mathfrak{a}, \forall \mathfrak{a} \in Z$.

$\left(\mathrm{P}_{10}\right): \phi$ is one-one map.

$\left(\mathrm{P}_{11}\right)$ : If $\phi$ is a regular map then it is identity.

$\left(\mathrm{P}_{12}\right)$ : If there exists an element $\mathfrak{a} \in Z$, such that $\phi(\mathfrak{a})=\mathfrak{a}$ then the map $\phi$ is identity.

$\left(\mathrm{P}_{13}\right)$ : If $\phi(\mathfrak{b}) * \mathfrak{a}=0$ or $\mathfrak{a} * \phi(\mathfrak{b})=0$ then $\phi(\mathfrak{b})=\mathfrak{a}, \forall \mathfrak{a}$, $\mathrm{b} \in Z$ i.e. $\phi$ is constant.

Proof $\left(\mathrm{P}_{9}\right)$ :

$$
\begin{gathered}
\phi(0)=\phi(\mathfrak{a} * \mathfrak{a}), \because \text { by }\left(\mathrm{P}_{3}\right) \\
=(\phi(\mathfrak{a}) * \mathfrak{a}) \wedge(\mathfrak{a} * \phi(\mathfrak{a})) \\
=(\mathfrak{a} * \phi(\mathfrak{a})) *[(\mathfrak{a} * \phi(\mathfrak{a})) *(\phi(\mathfrak{a}) * \mathfrak{a})] \\
=[(\mathfrak{a} * \phi(\mathfrak{a})) *(\mathfrak{a} * \phi(\mathfrak{a}))] *(\phi(\mathfrak{a}) * \mathfrak{a}), \because Z \text { is associative } \\
=0 *(\phi(\mathfrak{a}) * \mathfrak{a}), \because \text { by }\left(\mathrm{P}_{3}\right) \\
=(\phi(\mathfrak{a}) * \mathfrak{a}), \because \text { by }\left(\mathrm{P}_{1}\right)
\end{gathered}
$$

Proof $\left(\mathrm{P}_{10}\right)$ : Let $\mathfrak{a}, \mathfrak{b} \in Z$ such that

$$
\phi(\mathfrak{a})=\phi(\mathfrak{b})
$$

From $\left(\mathrm{P}_{9}\right)$ we have

$$
\phi(0)=\phi(\mathfrak{a}) * \mathfrak{a} \forall \mathfrak{a} \in Z,
$$

Also from $\left(\mathrm{P}_{9}\right)$ we have

$$
\phi(0)=\phi(\mathfrak{b}) * \mathfrak{b} \forall \mathfrak{b} \in Z
$$

From (2) and (3) we get

$$
\phi(\mathfrak{a}) * \mathfrak{a}=\phi(\mathfrak{b}) * \mathfrak{b}
$$

Using the result of equation (1) in equation (4) we get

$$
\phi(\mathfrak{a}) * \mathfrak{a}=\phi(\mathfrak{a}) * \mathfrak{b}
$$

By $\left(\mathrm{P}_{8}\right)$ left cancellation law holds in $Z$ therefore from (5) we get $\mathfrak{a}=\mathfrak{b}$. Hence $\phi$ is one to one.

Proof $\left(\mathrm{P}_{11}\right)$ : Let $\phi$ is regular then we have

$$
\phi(0)=0
$$


From $\left(\mathrm{P}_{9}\right)$ we have

$$
\phi(0)=\phi(\mathfrak{a}) * \mathfrak{a} \forall \mathfrak{a} \in Z
$$

From (6) and (7) we get

$$
\phi(\mathfrak{a}) * \mathfrak{a}=0 \forall \mathfrak{a} \in Z,
$$

Now by using $\left(\mathrm{P}_{3}\right)$ in the right hand side of equation (8) then (8) becomes

$$
\phi(\mathfrak{a}) * \mathfrak{a}=\mathfrak{a} * \mathfrak{a} \forall \mathfrak{a} \in Z, \because \mathfrak{a} * \mathfrak{a}=0
$$

By $\left(\mathrm{P}_{8}\right)$ right cancellation law holds in $Z$ therefore (9) becomes $\phi(\mathfrak{a})=\mathfrak{a} \forall \mathfrak{a} \in Z$.

Hence $\phi$ is the identity map.

Proof $\left(\mathrm{P}_{12}\right)$ : Let

$$
\phi(\mathfrak{a})=\mathfrak{a}
$$

Now by proposition $\left(\mathrm{P}_{7}\right)$ equation (10) is equivalent to

$$
\phi(\mathfrak{a}) * \mathfrak{a}=\mathfrak{a} * \mathfrak{a} \Rightarrow \phi(\mathfrak{a}) * \mathfrak{a}=0, \because \text { by }\left(\mathrm{P}_{3}\right)
$$

From $\left(\mathrm{P}_{9}\right)$ we have

$$
\phi(0)=\phi(\mathfrak{a}) * \mathfrak{a} \forall \mathfrak{a} \in Z
$$

So now using the result of equation (12) in the left hand side of equation (11) we get

$\phi(0)=0 \Longrightarrow \phi$ is regular which by $\left(P_{11}\right) \phi$ is the identity map.

Proof $\left(\mathrm{P}_{11}\right)$ : Let

$$
\phi(\mathfrak{b}) * \mathfrak{a}=0
$$

by proposition $\left(\mathrm{P}_{3}\right)$ equation (13) becomes

$$
\phi(\mathfrak{b}) * \mathfrak{a}=\mathfrak{a} * \mathfrak{a}
$$

by $\left(\mathrm{P}_{8}\right)$ right cancellation law holds in $Z$ therefore (14) becomes $\phi(\mathfrak{b})=\mathfrak{a}$.

Similarly for

$$
\mathfrak{a} * \phi(\mathfrak{b})=0
$$

by $\left(\mathrm{P}_{3}\right)$ equation (15) becomes

$$
\mathfrak{a} * \phi(\mathfrak{b})=\mathfrak{a} * \mathfrak{a}
$$

by $\left(\mathrm{P}_{8}\right)$ left cancellation law holds in $Z$ therefore (16) becomes $\phi(\mathfrak{b})=\mathfrak{a}$.

Proposition 3.7:- Let $\phi$ be a (Right, Left)-derivation of an associative PU-algebra $Z$ then

$\left(\mathrm{P}_{14}\right): \phi(0)=\mathfrak{a} * \phi(\mathfrak{a}), \forall \mathfrak{a} \in Z$

$\left(\mathrm{P}_{15}\right): \phi$ is one-one map.

$\left(\mathrm{P}_{16}\right)$ : If $\phi$ is a regular map then it is identity.

$\left(\mathrm{P}_{17}\right)$ : If there exists an element $\mathfrak{a} \in Z$, such that $\phi(\mathfrak{a})=\mathfrak{a}$ then the map

$\phi$ is identity.

$\left(\mathrm{P}_{18}\right)$ : If $\phi(\mathfrak{b}) * \mathfrak{a}=0$ or $\mathfrak{a} * \phi(\mathfrak{b})=0$ then $\phi(\mathfrak{b})=\mathfrak{a}, \forall \mathfrak{a}$, $\mathfrak{b} \in Z$ i.e. $\phi$ is constant.

Proof $\left(\mathrm{P}_{14}\right)$ :

$$
\phi(0)=\phi(\mathfrak{a} * \mathfrak{a}), \because \text { by }\left(\mathrm{P}_{3}\right)
$$

$$
\begin{gathered}
=(\mathfrak{a} * \phi(\mathfrak{a})) \wedge(\phi(\mathfrak{a}) * \mathfrak{a}) \\
=(\phi(\mathfrak{a}) * \mathfrak{a}) *[(\phi(\mathfrak{a}) * \mathfrak{a}) *(\mathfrak{a} * \phi(\mathfrak{a}))] \\
=[(\phi(\mathfrak{a}) * \mathfrak{a}) *(\phi(\mathfrak{a}) * \mathfrak{a})] *(\mathfrak{a} * \phi(\mathfrak{a})), \because Z \text { is associative } \\
=0 *(\mathfrak{a} * \phi(\mathfrak{a})), \because \text { by }\left(\mathrm{P}_{3}\right) \\
=\mathfrak{a} * \phi(\mathfrak{a}), \because \text { by }\left(\mathrm{P}_{1}\right)
\end{gathered}
$$

Proof $\left(\mathrm{P}_{15}\right)$ : Let $\mathfrak{a}, \mathfrak{b} \in Z_{\text {such that }}$

$$
\phi(\mathfrak{a})=\phi(\mathfrak{b})
$$

From $\left(\mathrm{P}_{14}\right)$ we have

$$
\phi(0)=\mathfrak{a} * \phi(\mathfrak{a}) \forall \mathfrak{a} \in Z
$$

Also from $\left(\mathrm{P}_{14}\right)$ we have

$$
\phi(0)=\mathfrak{b} * \phi(\mathfrak{b}) \forall \mathfrak{b} \in Z
$$

From (17) and (18) we get

$$
\mathfrak{a} * \phi(\mathfrak{a})=\mathfrak{b} * \phi(\mathfrak{b})
$$

Using (17) in the right hand side of equation (20) we get

$$
\mathfrak{a} * \phi(\mathfrak{a})=\mathfrak{b} * \phi(\mathfrak{a})
$$

By $\left(\mathrm{P}_{8}\right)$ right cancellation law holds in $Z$ therefore from (21) we get $\mathfrak{a}=\mathfrak{b}$ that is $\phi$ is one to one.

Proof $\left(\mathrm{P}_{16}\right)$ : Let $\phi$ is regular then we have

$$
\phi(0)=0
$$

From $\left(\mathrm{P}_{14}\right)$ we have

$$
\phi(0)=\mathfrak{a} * \phi(\mathfrak{a}) \forall \mathfrak{a} \in Z
$$

From (22) and (23) we get

$$
\mathfrak{a} * \phi(\mathfrak{a})=0 \forall \mathfrak{a} \in Z
$$

Now by using $\left(\mathrm{P}_{3}\right)$ in the right hand side of equation (24) then (24) becomes

$$
\mathfrak{a} * \phi(\mathfrak{a})=\mathfrak{a} * \mathfrak{a} \forall \mathfrak{a} \in Z \because \mathfrak{a} * \mathfrak{a}=0
$$

By $\left(\mathrm{P}_{8}\right)$ left cancellation law holds in $Z$ therefore (iv) becomes $\phi(\mathfrak{a})=\mathfrak{a}, \forall \mathfrak{a} \in Z$.

Hence $\phi$ is the identity map.

Proof $\left(\mathrm{P}_{17}\right)$ : Let

$$
\phi(\mathfrak{a})=\mathfrak{a}
$$

Now by proposition $\left(\mathrm{P}_{7}\right)$ equation (26) is equivalent to

$$
\mathfrak{a} * \phi(\mathfrak{a})=\mathfrak{a} * \mathfrak{a} \Longrightarrow \mathfrak{a} * \phi(\mathfrak{a})=0, \because \text { by }\left(P_{3}\right)
$$

From $\left(\mathrm{P}_{14}\right)$ we have

$$
\phi(0)=\mathfrak{a} * \phi(\mathfrak{a}) \forall \mathfrak{a} \in Z
$$

so now using (28) in (27) we get $\phi(0)=0 \Rightarrow \phi$ is regular which by $\left(\mathrm{P}_{16}\right) \phi$ is the identity map.

Proof $\left(\mathrm{P}_{18}\right)$ : 


$$
\text { Let } \phi(\mathfrak{b}) * \mathfrak{a}=0
$$

by proposition $\left(\mathrm{P}_{3}\right)$ the right hand side of equation (29) becomes

$$
\phi(\mathfrak{b}) * \mathfrak{a}=\mathfrak{a} * \mathfrak{a}
$$

by $\left(\mathrm{P}_{8}\right)$ right cancellation law holds in $Z$ therefore (30) becomes $\phi(\mathfrak{b})=\mathfrak{a}$

Similarly

$$
\mathfrak{a} * \phi(\mathfrak{b})=0
$$

by $\left(\mathrm{P}_{3}\right)$ equation (31) becomes,

$$
\mathfrak{a} * \phi(\mathfrak{b})=\mathfrak{a} * \mathfrak{a}
$$

by $\left(\mathrm{P}_{8}\right)$ left cancellation law holds in $Z$ therefore (32) becomes $\phi(\mathfrak{b})=\mathfrak{a}$.

Theorem 3.8: Let $Z$ is an associative PU-algebra

$\left(\mathrm{P}_{\mathrm{a}}\right)$ : If $\phi$ is (Left, Right) -regular derivation of $Z$ then $\phi(\mathfrak{a})=\mathfrak{a} \wedge \phi(\mathfrak{a}) \forall \mathfrak{a} \in Z$.

$\left(\mathrm{P}_{\mathrm{b}}\right)$ : If $\phi$ is (Right, Left) -regular derivation of $Z$ then $\phi(\mathfrak{a})=\phi(\mathfrak{a}) \wedge \mathfrak{a} \forall \mathfrak{a} \in Z$.

Proof $\left(\mathrm{P}_{\mathrm{a}}\right)$ : Since $\phi$ is regular therefore we have

$$
\phi(0)=0
$$

Now consider for some $\mathfrak{a} \in Z$, we have

$$
\begin{gathered}
\phi(\mathfrak{a})=\phi(0 * \mathfrak{a}), \because \text { by }\left(\mathrm{P}_{1}\right) \\
=(\phi(0) * \mathfrak{a}) \wedge(0 * \phi(\mathfrak{a})), \because \text { by definition } 3.1 \\
=(0 * \mathfrak{a}) \wedge(0 * \phi(\mathfrak{a})), \because \text { by using }(33) \\
=\mathfrak{a} \wedge \phi(\mathfrak{a}), \because \text { by }\left(\mathrm{P}_{1}\right)
\end{gathered}
$$

Proof $\left(\mathrm{P}_{\mathrm{b}}\right)$ : Since $\phi$ is regular therefore we have

$$
\phi(0)=0
$$

Now consider for some $\in Z, \phi(\mathfrak{a})=\phi(0 * \mathfrak{a}), \because$ by $\left(\mathrm{P}_{1}\right)$

$=(0 * \phi(\mathfrak{a})) \wedge(\phi(0) * \mathfrak{a}) \because$ by definition 3.1

$=(0 * \phi(\mathfrak{a})) \wedge(0 * \mathfrak{a}) \because$ by using $(34)$

$$
=\phi(\mathfrak{a}) \wedge \mathfrak{a} \because \text { by }\left(\mathrm{P}_{1}\right)
$$

Theorem 3.9: Let $\phi$ is a self-map of an associative PUAlgebra $Z$ then $(\mathfrak{a} *(\mathfrak{a} * \phi(\mathfrak{a}))) * \mathfrak{a}=(\phi(\mathfrak{a}) *(\phi(\mathfrak{a}) * \mathfrak{a})) * \mathfrak{a}$.

Proof: Since by (theorem 3.8 $\left(\mathrm{P}_{\mathrm{b}}\right)$ ) we have,

$$
\phi(\mathfrak{a})=\phi(\mathfrak{a}) \wedge \mathfrak{a}=\mathfrak{a} *(\mathfrak{a} * \phi(\mathfrak{a}))
$$

By $\left(\mathrm{P}_{7}\right)$ equation (35) is equivalent to

$$
\phi(\mathfrak{a}) * \mathfrak{a}=(\mathfrak{a} *(\mathfrak{a} * \phi(\mathfrak{a}))) * \mathfrak{a}
$$

on the other hand from (theorem $3.8\left(\mathrm{P}_{\mathrm{a}}\right)$ ) we have

$$
\phi(\mathfrak{a})=\mathfrak{a} \wedge \phi(\mathfrak{a})=\phi(\mathfrak{a}) *(\phi(\mathfrak{a}) * \mathfrak{a})
$$

Similarly by $\left(\mathrm{P}_{7}\right)$ equation (37) is equivalent to

$$
\phi(\mathfrak{a}) * \mathfrak{a}=(\phi(\mathfrak{a}) *(\phi(\mathfrak{a}) * \mathfrak{a})) * \mathfrak{a}
$$

from (36) and (38) we get

$$
(\mathfrak{a} *(\mathfrak{a} * \phi(\mathfrak{a}))) * \mathfrak{a}=(\phi(\mathfrak{a}) *(\phi(\mathfrak{a}) * \mathfrak{a})) * \mathfrak{a} .
$$

Theorem 3.10: If $\phi$ is a derivation on an associative PUalgebra $Z$ then $\forall \mathfrak{a} \in Z$,

$$
\begin{aligned}
& \left(\mathrm{P}_{\mathrm{c}}\right): \phi(\mathfrak{a} * \phi(\mathfrak{a}))=0 \\
& \left(\mathrm{P}_{\mathrm{d}}\right): \phi(\phi(\mathfrak{a}) * \mathfrak{a})=0
\end{aligned}
$$

Proof $\left(\mathrm{P}_{\mathrm{c}}\right)$ : Let $\phi$ is a (Left, Right)-derivation on $Z$ then

$$
\begin{gathered}
\phi(\mathfrak{a} * \phi(\mathfrak{a}))=(\phi(\mathfrak{a}) * \phi(\mathfrak{a})) \wedge(\mathfrak{a} * \phi(\phi(\mathfrak{a})))=0 \wedge(\mathfrak{a} * \\
\phi(\phi(\mathfrak{a}))), \because \text { by using }\left(\mathrm{P}_{3}\right) \\
\Rightarrow \phi(\mathfrak{a} * \phi(\mathfrak{a}))=(\mathfrak{a} * \phi(\phi(\mathfrak{a}))) *[(\mathfrak{a} * \phi(\phi(\mathfrak{a}))) * 0](
\end{gathered}
$$

As $Z$ is an associative PU-algebra therefore we can write equation (39) as

$\phi(\mathfrak{a} * \phi(\mathfrak{a}))=[\mathfrak{a} * \phi(\phi(\mathfrak{a}))) *(\mathfrak{a} * \phi(\phi(\mathfrak{a}))] * 0=0 * 0=0$, $\because$ by using $\left(\mathrm{P}_{3}\right)$

Proof $\left(\mathrm{P}_{\mathrm{d}}\right)$ : Let $\phi$ is a (Right, Left)-derivation on $Z$ then

$$
\begin{gathered}
\phi(\phi(\mathfrak{a}) * \mathfrak{a})=(\phi(\mathfrak{a}) * \phi(\mathfrak{a})) \wedge(\phi(\phi(\mathfrak{a})) * \mathfrak{a})=0 \wedge(\phi(\phi(\mathfrak{a})) * \\
\mathfrak{a}), \because \text { by using }\left(\mathrm{P}_{3}\right) \\
\Rightarrow \phi(\phi(\mathfrak{a}) * \mathfrak{a})=(\phi(\phi(\mathfrak{a})) * \mathfrak{a}) *[(\phi(\phi(\mathfrak{a})) * \mathfrak{a}) * 0](40)
\end{gathered}
$$

As $Z$ is an associative PU-algebra therefore (39) can be written as

$\phi(\phi(\mathfrak{a})) * \mathfrak{a})=[(\phi(\phi(\mathfrak{a})) * \mathfrak{a}) *(\phi(\phi(\mathfrak{a})) * \mathfrak{a})] * 0=0 * 0=0, \because$ by using $\left(\mathrm{P}_{3}\right)$

Theorem 3.11: Let $\phi$ is a regular (Left, Right)-derivation of an associative PU-algebra $Z$ then the following results hold in $Z$.

$$
\begin{gathered}
\left(\mathrm{P}_{\mathfrak{e}}\right): \phi(\mathfrak{a})=\mathfrak{a} \\
\left(\mathrm{P}_{\mathrm{f}}\right): \phi(\mathfrak{a}) * \mathfrak{b}=\mathfrak{a} * \phi(\mathfrak{b}) \forall \mathfrak{a}, \mathfrak{b} \in Z \\
\left(\mathrm{P}_{\mathfrak{g}}\right): \phi(\mathfrak{b} * \mathfrak{a})=\phi(\mathfrak{a}) * \mathfrak{b}=\phi(\mathfrak{a}) * \phi(\mathfrak{b})=\mathfrak{a} * \phi(\mathfrak{b}) \\
\left(\mathrm{P}_{\mathfrak{h}}\right): \operatorname{Ker}(\phi)=\{\mathfrak{a} \in Z ; \phi(\mathfrak{a})=0\} \text { is a subalgebra of } Z . \\
\text { Proof }\left(\mathrm{P}_{\mathrm{e}}\right): \phi(\mathfrak{a})=\phi(0 * \mathfrak{a}), \\
\because \text { by using }\left(\mathrm{P}_{1}\right) \\
=(\phi(0) * \mathfrak{a}) \wedge(0 * \phi(\mathfrak{a})) \\
=(0 * \mathfrak{a}) \wedge(0 * \phi(\mathfrak{a})), \because \phi \text { is regular } \\
=\mathfrak{a} \wedge \phi(\mathfrak{a}), \because \text { by using }\left(\mathrm{P}_{1}\right) \\
=\phi(\mathfrak{a}) *(\phi(\mathfrak{a}) * \mathfrak{a}) \\
=(\phi(\mathfrak{a}) * \phi(\mathfrak{a})) * \mathfrak{a}, \because Z \text { is associative } \\
=0 * \mathfrak{a}=\mathfrak{a} \because \text { by using }\left(\mathrm{P}_{1}\right)
\end{gathered}
$$

Hence $\phi(\mathfrak{a})=\mathfrak{a}$.

Proof $\left(\mathrm{P}_{\mathrm{f}}\right)$ : As $\phi$ is a regular (Left, Right)-derivation of an 
associative PU-algebra $Z$ then by $\left(\mathrm{P}_{\mathrm{e}}\right)$ we have

$$
\phi(\mathfrak{a})=\mathfrak{a}, \forall \mathfrak{a} \in Z
$$

And

$$
\phi(\mathfrak{b})=\mathfrak{b}, \forall \mathfrak{b} \in Z
$$

By using the results of equations (41) and (42) we get $\phi(\mathfrak{a}) * \mathfrak{b}=\mathfrak{a} * \mathfrak{b}=\mathfrak{a} * \phi(\mathfrak{b})$.

Proof $\left(\mathrm{P}_{\mathrm{g}}\right)$ : As $\phi$ is a regular (Left, Right)-derivation of an associative PU-algebra $Z$ then by $\left(\mathrm{P}_{\mathrm{e}}\right)$ we have

$$
\phi(\mathfrak{a})=\mathfrak{a}, \forall \mathfrak{a} \in Z
$$

Therefore for any $\mathfrak{a}, \mathfrak{b} \in Z$,

$$
\begin{gathered}
\text { We have } \phi(\mathfrak{a} * \mathfrak{b})=\mathfrak{a} * \mathfrak{b} \because \text { by using }\left(\mathrm{P}_{\mathrm{e}}\right) \\
\Rightarrow \phi(\mathfrak{a} * \mathfrak{b})=\phi(\mathfrak{a}) * \phi(\mathfrak{b}), \because \text { by equation }(43) \\
\Rightarrow \phi(\mathfrak{a} * \mathfrak{b})=\phi(\mathfrak{a}) * \mathfrak{b}, \because \text { by equation }(43) \\
\text { And } \phi(\mathfrak{a} * \mathfrak{b})=\mathfrak{a} * \phi(\mathfrak{b}) \because \text { by equation }(43)
\end{gathered}
$$

The equations (45), (46) and (47) imply that

$$
\begin{aligned}
& \phi(\mathfrak{b} * \mathfrak{a})=\phi(\mathfrak{a}) * \mathfrak{b}=\phi(\mathfrak{a}) * \phi(\mathfrak{b})=\mathfrak{a} * \phi(\mathfrak{b}) \\
& \text { Proof }\left(\mathrm{P}_{\mathrm{h}}\right) \text { : Let } \mathfrak{a}, \mathfrak{b} \in \operatorname{Ker}(\phi) \text { then } \phi(\mathfrak{a})=0
\end{aligned}
$$

And

$$
\phi(\mathfrak{b})=0
$$

As $\phi$ is a regular derivation therefore from $\left(\mathrm{P}_{\mathrm{g}}\right)$ we have,

$$
\phi(\mathfrak{a} * \mathfrak{b})=\phi(\mathfrak{a}) * \phi(\mathfrak{b})
$$

Using (48) and (49) in the right hand side of equation (50) we get $\phi(\mathfrak{a} * \mathfrak{b})=0$

$$
\Longrightarrow \mathfrak{a} * \mathfrak{b} \in \operatorname{Ker}(\phi) \Longrightarrow \operatorname{Ker}(\phi) \text { is a subalgebra of } \boldsymbol{Z} \text {. }
$$

\section{Conclusion}

We see that derivations with special properties play a central role in the investigation of the structure of an algebraic system.

The forthcoming study of derivations in PU-algebras may be the following topics are worth to be taken into account.

To describe left derivations in PU-algebras and investigate a regular left derivations by using this concept.

To introduce the concept of f-derivations, $t$-derivations, $t$ bi-derivations and $(\alpha, \beta)$-derivations in PU-algebras.

To refer this concept to some other algebraic structures.

To consider the results of this concept to some possible applications in information systems and computer sciences.

\section{References}

[1] Iséki, K. (1966). An algebra related with a propositional calculus. Proceedings of the Japan Academy, 42 (1), 26-29.
[2] Iséki, K. (1978). An introduction to the theory of BCKalgebras. Math. Japonica, 23, 1-26.

[3] Imai, Y., \& Iseki, K. (1966). On axioms of Proportional calculi xiv proc. Japan Acad, 42, 19-22.

[4] Neggers, J., Ahn, S. S., \& Kim, H. S. (2001). On Q- algebras. International Journal of Mathematics and Mathematical Sciences, 27 (12), 749-757.

[5] Megalai, K., \& Tamilarasi, A. (2010). Classification of TMalgebra. IJCA Special Issue on "Computer Aided Soft Computing Techniques for Imaging and Biomedical Applications" CASCT.

[6] Mostafa, Samy M., MA Abdel Naby, and A. I. Elkabany. "New view of ideals on PU-algebra." International Journal of Computer Applications 111.4, 18 pages, (2015). View at: Publisher Site | Google Scholar.

[7] Hvala, B. (1998). Generalized derivations in rings. Communications in Algebra, 26 (4), 1147-1166.

[8] Bell, H. E., \& Kappe, L. C. (1989). Rings in which derivations satisfy certain algebraic conditions. Acta Mathematica Hungarica, 53 (3-4), 339-346.

[9] Bell, H. E., \& Mason, G. (1987). On derivations in near- rings. In North-Holland Mathematics Studies (Vol. 137, pp. 31-35). North-Holland.

[10] Brešar, M., \& Vukman, J. (1990). On left derivations and related mappings. Proceedings of the American Mathematical Society, 110 (1), 7-16.

[11] Bresar, M. (1991). On the distance of the composition of two derivations to the generalized derivations. Glasgow Mathematical Journal, 33 (1), 89-93.

[12] Muhiuddin, G., \& Al-Roqi, A. M. (2012). On-Derivations in BCI-Algebras. Discrete Dynamics in Nature and Society, 2012.

[13] Ilbira, S., Firat, A., \& Jun, Y. B. (2011). On symmetric biderivations of BCI-algebras. Applied Mathematical Sciences, 5 (57-60), 2957-2966.

[14] Al-Kadi, D. (2017). On (f, g)-Derivations of G-Algebras.

[15] Zhao, W. (2018). Some open problems on locally finite or locally nilpotent derivations and $\varepsilon$-derivations. Communications in Contemporary Mathematics, 20 (04), 1750056 .

[16] Alekseev, A. V., \& Arutyunov, A. A. (2020). Derivations in semigroup algebras. Eurasian Mathematical Journal, 11 (2), $9-18$.

[17] Muhiuddin, G., \& Al-Roqi, A. M. (2012). On $(\alpha, \beta)$ derivations in BCI-algebras. Discrete Dynamics in Nature and Society, 2012.

[18] Abujabal, H. A., \& Al-Shehri, N. O. (2017). On left derivations of BCI-algebras. Soochow Journal of Mathematics, $33(3), 435$. 